\title{
OPERATIONS WITH SLICELY COUNTABLY DETERMINED SETS
}

\author{
VLADIMIR KADETS, ANTONIO PÉREZ, AND DIRK WERNER
}

\author{
Dedicated to the memory of Paweł Domański
}

\begin{abstract}
The notion of slicely countably determined (SCD) sets was introduced in 2010 by A. Avilés, V. Kadets, M. Martín, J. Merí and $\mathrm{V}$. Shepelska. We solve in the negative some natural questions about preserving being SCD by the operations of union, intersection and Minkowski sum. Moreover, we demonstrate that corresponding examples exist in every space with the Daugavet property and can be selected to be unit balls of some equivalent norms. We also demonstrate that almost SCD sets need not be SCD, thus answering a question posed by A. Avilés et al.
\end{abstract}

\section{INTRODUCTION}

The property "slicely countably determined" (SCD for short) for Banach spaces and their subsets was first considered and studied in [1] (see [2] for the complete version), proving to have noticeable applications to Banach spaces with the Daugavet property, numerical index one and other related properties $[2,7,9,10]$.

Let us recall the basic definition and examples. In this paper we use the letters $X, Y$ and $E$ to denote Banach spaces. By a slice of a subset $A \subset X$ we mean a non-empty set which is the intersection of $A$ with an open half-space. In other words, it is a set of the form

$$
S\left(A, x^{*}, \varepsilon\right)=\left\{x \in A: x^{*}(x)>\sup _{a \in A} x^{*}(a)-\varepsilon\right\},
$$

where $x^{*}: X \rightarrow \mathbb{R}$ is a non-zero real linear bounded functional. In the case of $x^{*}=0$ the above definition also makes sense and gives the degenerate slice $S(A, 0, \varepsilon)=A$.

Definition 1.1 ([2]). Let $X$ be a Banach space, $A \subset X$ be a bounded subset. A sequence of non-empty subsets $U_{n} \subset A$ is called determining if for each $B \subset X$ that intersects all the $U_{n}, n \in \mathbb{N}$, it holds that $A \subset \overline{\operatorname{conv}}(B)$. The set $A$ is said to be $S C D$ if there is a determining sequence of slices of $A$. The

The research of the first author is done in the framework of the Ukrainian Ministry of Science and Education Research Program 0115U000481, and it was partially done during his stay in Murcia under the support of MINECO/FEDER project MTM2014-57838-C21-P, partially during his visits to the University of Granada which were supported by the Spanish MINECO/FEDER project MTM2015-65020-P, and partially during his visit to Freie Universität Berlin in the framework of a grant from the Alexander-von-Humboldt Stiftung. The second author was partially supported by the MINECO/FEDER project MTM2014-57838-C2-1-P and a Ph.D. fellowship of "La Caixa Foundation". 
space $X$ is said to be an $S C D$ space ( $X \in \mathrm{SCD}$ for short) whenever all its bounded subsets are SCD.

Note that every SCD set is separable. Let us further remark that in the definition of SCD sets one may also permit that some slices are degenerate ones.

We will use several times the following consequence of the Hahn-Banach theorem, remarked first for convex sets in [2, Proposition 2.2]:

Lemma 1.2. Let $U \subset X$ be a bounded set. A sequence $\left\{V_{n}: n \in \mathbb{N}\right\}$ of nonempty subsets of $U$ is determining for $U$ if and only if it has the following property (*): every slice of $U$ contains one of the $V_{n}$.

Proof. Let $(*)$ be fulfilled and let $B \subset U$ intersect all the $V_{n}$. Then $B$ intersects all the slices of $U$, and then by the Hahn-Banach separation theorem $\overline{\operatorname{conv}}(B) \supset U$. Now the "only if" part. Assume that some slice $S=S\left(U, x^{*}, \varepsilon\right)$ of $U$ does not contain any of the $V_{n}$. Then $U \backslash S$ intersects all the $V_{n}$. But $U \backslash S \subset\left\{x \in X: x^{*}(x) \leq \sup _{a \in U} x^{*}(a)-\varepsilon\right\}$, hence $\overline{\operatorname{conv}}(U \backslash S) \subset\left\{x \in X: x^{*}(x) \leq \sup _{a \in U} x^{*}(a)-\varepsilon\right\}$ which means that $\overline{\operatorname{conv}}(U \backslash S) \not \supset U$, and consequently $\left\{V_{n}: n \in \mathbb{N}\right\}$ is not determining.

We can restrict ourselves to study bounded, closed and convex sets because of the following result ([9, Proposition 7.20] and [2, Remark 2.7]). Since we are going to use it several times, we will sketch the proof for the readers' convenience.

Lemma 1.3. Let $X$ be a Banach space. A bounded set $A \subset X$ is $S C D$ if and only if its convex hull $\operatorname{conv}(A)$ is $S C D$, and if and only if its closed convex hull $\overline{\operatorname{conv}}(A)$ is $S C D$.

Proof. It follows readily from the definition that a bounded set is SCD if and only if its closure is, and that $A$ is SCD once its convex hull is; cf. [2, Remark 2.7]. Suppose now that $A$ is $\mathrm{SCD}$, and let $\left\{S\left(A, x_{n}^{*}, \varepsilon_{n}\right): n \in \mathbb{N}\right\}$ be a family of slices determining for $A$. We consider the following (countable) family of slices of $\operatorname{conv}(A)$ :

$$
\mathcal{S}:=\left\{S\left(\operatorname{conv}(A), x_{n}^{*}, \varepsilon_{n} / k\right): n, k \in \mathbb{N}\right\} .
$$

Given any slice $S\left(\operatorname{conv}(A), x^{*}, \varepsilon\right)$ of $\operatorname{conv}(A)$, where $\left\|x^{*}\right\|=1$ without loss of generality, we will show that it contains an element of $\mathcal{S}$, thus proving that $\operatorname{conv}(A)$ is SCD by Lemma 1.2. Now, for the slice of $A$ given by $S\left(A, x^{*}, \varepsilon / 2\right)$ we know that there is $n_{0} \in \mathbb{N}$ such that $S\left(A, x_{n_{0}}, \varepsilon_{n_{0}}\right) \subset S\left(A, x^{*}, \varepsilon / 2\right)$. Taking $k \in \mathbb{N}$ big enough we will argue that

$$
S\left(\operatorname{conv}(A), x_{n_{0}}^{*}, \varepsilon_{n_{0}} / k\right) \subset \operatorname{conv} S\left(A, x_{n_{0}}^{*}, \varepsilon_{n_{0}}\right)+\frac{\varepsilon}{2} B_{X} .
$$

To prove this inclusion we let $r:=\sup _{a \in A} x_{n_{0}}^{*}(a)$ and $M:=\sup _{a \in A}\|a\|$, hence also $\sup _{a \in \operatorname{conv}(A)} x_{n_{0}}^{*}(a)=r$. Consider a convex combination $a=$ $\sum_{i=1}^{n} \lambda_{i} a_{i}$ of elements $a_{i} \in A$ such that $x_{n_{0}}^{*}(a)>r-\varepsilon_{n_{0}} / k$ where $k$ is not yet specified. Let $I=\left\{i: x_{n_{0}}^{*}\left(a_{i}\right)>r-\varepsilon_{n_{0}}\right\}$ and $J=\left\{i: x_{n_{0}}^{*}\left(a_{i}\right) \leq r-\varepsilon_{n_{0}}\right\}$. We then have

$$
r-\frac{\varepsilon_{n_{0}}}{k}<\sum_{i \in I} \lambda_{i} x_{n_{0}}^{*}\left(a_{i}\right)+\sum_{i \in J} \lambda_{i} x_{n_{0}}^{*}\left(a_{i}\right) \leq r \sum_{i \in I} \lambda_{i}+\sum_{i \in J} \lambda_{i}\left(r-\varepsilon_{n_{0}}\right),
$$


which implies

$$
\sum_{i \in J} \lambda_{i}<\frac{1}{k} \quad \text { and } \quad \Lambda:=\sum_{i \in I} \lambda_{i}>1-\frac{1}{k} .
$$

Now put $\mu_{i}:=\lambda_{i} / \Lambda$ for $i \in I$ and consider the element

$$
a^{\prime}=\sum_{i \in I} \mu_{i} a_{i} \in \operatorname{conv} S\left(A, x_{n_{0}}^{*}, \varepsilon_{n_{0}}\right) .
$$

The estimate

$$
\left\|a-a^{\prime}\right\|=\left\|(\Lambda-1) \sum_{i \in I} \mu_{i} a_{i}+\sum_{i \in J} \lambda_{i} a_{i}\right\| \leq|\Lambda-1| M+\sum_{i \in J} \lambda_{i} M<\frac{2 M}{k}
$$

shows that the above inclusion holds true whenever $k \geq 4 M / \varepsilon$.

It now follows for this choice of $k$ that

$$
\begin{aligned}
S\left(\operatorname{conv}(A), x_{n_{0}}^{*}, \varepsilon_{n_{0}} / k\right) & \subset \operatorname{conv} S\left(A, x_{n_{0}}^{*}, \varepsilon_{n_{0}}\right)+\frac{\varepsilon}{2} B_{X} \\
& \subset \operatorname{conv} S\left(A, x^{*}, \varepsilon / 2\right)+\frac{\varepsilon}{2} B_{X} \\
& \subset S\left(\operatorname{conv}(A), x^{*}, \varepsilon / 2\right)+\frac{\varepsilon}{2} B_{X} .
\end{aligned}
$$

Since trivially $S\left(\operatorname{conv}(A), x_{n_{0}}^{*}, \varepsilon_{n_{0}} / k\right) \subset \operatorname{conv}(A)$, we finally get

$$
\begin{aligned}
S\left(\operatorname{conv}(A), x_{n_{0}}^{*}, \varepsilon_{n_{0}} / k\right) & \subset\left(S\left(\operatorname{conv}(A), x^{*}, \varepsilon / 2\right)+\frac{\varepsilon}{2} B_{X}\right) \cap \operatorname{conv}(A) \\
& \subset S\left(\operatorname{conv}(A), x^{*}, \varepsilon\right) .
\end{aligned}
$$

In the case of convex sets a well-known result of Bourgain lets us replace the sequence of slices by a sequence of relatively weakly open subsets or even by a sequence of convex combinations of slices in the definition of an SCD set [2, Proposition 2.18]. A non-convex set can be not SCD, and still possess a determining sequence of relatively weakly open subsets; this will be proved in Proposition 2.6 below.

Proposition 1.4. The following conditions are sufficient for a convex, bounded and separable subset $A \subset X$ to be $S C D$, see [2, Section 2] for details:

(i) $A$ is Asplund, i.e., $\left(X^{*}, \rho_{A}\right)$ is separable where $\rho_{A}\left(x^{*}\right)=\sup _{a \in A}\left|x^{*}(a)\right|$ for each $x^{*} \in X^{*}$.

(ii) $A$ is huskable, i.e., $A$ is the closed convex hull of all $a \in A$ satisfying that for each $\varepsilon>0$ there is a relatively weakly open set $W \subset A$ with diameter less than $\varepsilon$ containing a (immediate consequence of $[2$, Theorem 2.19]). In particular, this happens if $A$ is dentable.

(iii) $A$ is strongly regular, i.e., every convex subset $L \subset A$ has convex combinations of slices of arbitrarily small diameter.

(iv) $\left(A, \sigma\left(A, X^{*}\right)\right)$ has a countable $\pi$-basis, that is, a countable family of relatively weakly open non-empty subsets such that each relatively weakly open non-empty subset of A contains a member of that family. This is the case, in particular, if $A$ does not contain $\ell_{1}$-sequences.

As a consequence of the previous examples, if $X$ is a separable Banach space without isomorphic copies of $\ell_{1}$ (in particular if it is Asplund) or if it has the convex point of continuity property $(\mathrm{CPCP})$, in particular if it has the Radon-Nikodym property (RNP), then $X \in \mathrm{SCD}$. 
Another class of examples [7, Theorem 3.1]: the unit ball of every space with a 1-unconditional basis is SCD. It is an open question whether every Banach space with an unconditional basis is an SCD space.

In order to present typical applications of SCD sets to operators in Banach spaces, let us introduce some definitions. A bounded linear operator $T: X \rightarrow$ $X$ satisfies the Daugavet equation if

$$
\|I+T\|=1+\|T\|,
$$

and satisfies the alternative Daugavet equation if

$$
\max \{\|I+\theta T\|:|\theta|=1\}=1+\|T\| .
$$

A Banach space has the Daugavet property if every rank-1 operator $T: X \rightarrow$ $X$ satisfies the Daugavet equation, and it possesses the alternative Daugavet property if every rank-1 operator $T: X \rightarrow X$ satisfies the alternative Daugavet equation. Typical examples of spaces possessing the Daugavet property (and consequently the alternative Daugavet property) are $C[0,1]$ and $L_{1}[0,1]$. Typical examples of spaces not possessing the Daugavet property but nevertheless having the alternative Daugavet property are $c_{0}$ and $\ell_{1}$. See [11] and [13].

Theorem 4.4 of [2] says, in particular, that if $X$ possesses the alternative Daugavet property and $B_{X}$ is SCD, then every bounded linear operator on $X$ satisfies the alternative Daugavet equation. Theorem 5.3 and Proposition 5.8 of [2] say that if $X$ possesses the (alternative) Daugavet property and $T\left(B_{X}\right)$ is $\mathrm{SCD}$, then the operator $T$ satisfies the (alternative) Daugavet equation. More applications in the same vein can be found in [3, Section 3], [8, Theorems 3.4 and 3.7] and [9, Sections 3, 7].

The number of known examples of separable Banach spaces $X$ that are not SCD is limited to those having the Daugavet property. In these spaces the unit ball satisfies following "anti-SCD" condition, see [2, Example 2.13].

Lemma 1.5. Let $X$ be a Banach space with the Daugavet property. Then, for every sequence of slices $\left(S_{n}\right)$ of $B_{X}$ and every $x \in S_{X}$ there is a set $B=\left\{x_{n}: n \in \mathbb{N}\right\}$ with $x_{n} \in S_{n}, n=1,2, \ldots$, such that $x \notin \operatorname{span} B$.

It is an open question whether every separable Banach space $X \notin \mathrm{SCD}$ is isomorphic to a space with the Daugavet property.

When studying applications of the property SCD, it was pointed out in $[2$, Remark 4.5 and 5.4] that some of them (in particular the above-mentioned [2, Theorem 4.4, Theorem 5.3]) hold if in the definition of an SCD set we replace the convex hull by the absolutely convex hull, introducing the next presumably weaker condition:

Definition 1.6. A bounded set $A \subset X$ of a Banach space $X$ is called almost $S C D$ (aSCD, in short) if there is a sequence of slices $S_{n}$ of $A$ satisfying that for each $B \subset X$ with $B \cap S_{n} \neq \emptyset$ for every $n \in \mathbb{N}$, it holds that $A \subset \overline{\operatorname{aconv}}(B)$.

It is asked in [2, Question 7.5] whether the classes of aSCD sets and SCD sets coincide. In the present paper we demonstrate that the properties aSCD and SCD are not equivalent for general bounded closed convex sets, but in the case that is most important for the applications, namely the case of balanced bounded closed convex sets, the equivalence holds true. We also solve in the 
negative natural questions about preserving SCD by the operations of union, intersection and Minkowski sum.

The main part of the paper consists of four sections. At the beginning of Section 2 we construct a set $A$, whose properties will be the base of all the remaining examples (the letter $A$ will be fixed afterwards for that special set). Then, we present the promised examples for the intersection of SCD sets (which will be $A$ and $-A$ ), after that for the Minkowski sum (which will again be $A$ and $-A$ ), and finally for the union (some shifts of $A$ and $-A$ ). In fact, we demonstrate the existence of such examples in every space with the Daugavet property. The examples constructed in Section 2 are not centrally symmetric, which is not entirely satisfactory, because in all the applications mentioned above only sets symmetric with respect to zero appear. Section 3 is devoted to the symmetrization of our examples, after which one can see that the operations of Minkowski sum, union and intersection do not preserve the property SCD even if the sets in question are unit balls of some equivalent norms. In Section 4 we give an example of an aSCD set that is not SCD, which will be the union of $A$ with a specially constructed subset of $-A$. The last short section lists some open problems about SCD sets.

Since in the definition of a slice and, consequently, in the definition of an SCD space only real scalars are used, below, if the contrary is not stated explicitly, we will consider only real Banach spaces. We have already used without explanation some standard Banach space notation like $B_{X}, S_{X}$ or $X^{*}$ for the closed unit ball, unit sphere and the dual space respectively. All unexplained notation below (if any) is also standard and can be found in every Banach space textbook, for example in [5].

\section{The PROMised EXAMPLES}

2.1. The intersection of SCD sets. The examples which we are going to present in this paper will be constructed in an arbitrary Banach space $X$ with the Daugavet property. According to [12, Theorem 4.5] $X$ contains a separable subspace with the Daugavet property, so without loss of generality we assume that $X$ itself is separable. Fix a one-codimensional closed subspace $E \subset X$. According to [11, Theorem 2.14] $E$ also has the Daugavet property, so $B_{E}$ enjoys the property from Lemma 1.5 , consequently $B_{E}$ is neither SCD, nor aSCD. The aim of the construction below is to include $B_{E}$ into an SCD set $A \subset X$ in such a way that $B_{E}$ lies in the boundary of $A$. This construction will be used in all the examples presented in this paper.

Recall that a space $Y$ is called locally uniformly rotund or locally uniformly convex (LUR for short) if for every $y \in S_{Y}$ and every sequence $\left(y_{n}\right)$ in $B_{Y}$ the condition $\left\|y+y_{n}\right\| \rightarrow 2$ implies that $\left\|y-y_{n}\right\| \rightarrow 0$. In a LUR space $Y$ every point $y$ of the unit sphere $S_{Y}$ is strongly exposed, that is, there is $y^{*} \in S_{Y^{*}}$ with $y^{*}(y)=1$ such that every sequence $\left(y_{n}\right)$ in $B_{Y}$ with $y^{*}\left(y_{n}\right) \rightarrow y^{*}(y)$ satisfies that $\left\|y-y_{n}\right\| \rightarrow 0$. It is a classical result by M. Kadets ([6], see also [5, p. 383, Theorem 8.1]) that every separable Banach space admits an equivalent LUR norm.

In fact, there is an equivalent LUR norm $\varphi: E \rightarrow[0,+\infty)$ such that $\frac{1}{2}\|x\| \leq \varphi(x) \leq\|x\|$ for all $x \in E$. Then for every $t>0$ the formula

$$
\|x\|_{t}=\sqrt{\|x\|^{2}+t^{2} \varphi(x)^{2}}, \quad x \in E
$$


defines an equivalent LUR norm on $E$ [4, Chapter 2, p. 53, beginning of Section 2] satisfying that

$$
\|x\| \leq\|x\|_{t} \leq \sqrt{1+t^{2}}\|x\| .
$$

In particular every point of the unit sphere $S_{\left(E,\|\cdot\|_{t}\right)}$ is strongly exposed. If $t=0$, then we get the original norm on $E$, i.e., $\|x\|_{0}=\|x\|$. We are going to use the notation $\|\cdot\|_{t}^{*}$ for the norm of $\left(E,\|\cdot\|_{t}\right)^{*}$. In the case of $t=0$, where $\|\cdot\|_{0}$ is just the original norm $\|\cdot\|$, we will write $\left\|y^{*}\right\|_{0}^{*}=\left\|y^{*}\right\|$.

We now construct the set which plays the fundamental role in all our counterexamples. Let $e_{0} \in X \backslash E$ be a fixed element of norm 1. Then $X=E \oplus \operatorname{span} e_{0}$. In the sequel we will use notation $x \oplus t$ in order to denote an element of the form $x+t e_{0}$, where $x \in E, t \in \mathbb{R}$. We will also consider the following equivalent norm on $X:\|x \oplus t\|_{\infty}=\max \{\|x\|,|t|\}$. Remark that the dual space to our $X=E \oplus \operatorname{span} e_{0}$ can be represented as the set of formal expressions $y^{*} \oplus \lambda, y^{*} \in E^{*}, \lambda \in \mathbb{R}$, that act on elements of $X$ by the natural rule $\left\langle y^{*} \oplus \lambda, x \oplus t\right\rangle=y^{*}(x)+\lambda t$.

Proposition 2.1. The subset

$$
A:=\left\{x \oplus t \in X:\|x\|_{t}^{2}+3 t^{2} \leq 1, t \geq 0\right\} \subset X
$$

has the following properties:

(a) Every element $x \oplus t \in A$ satisfies $t \in\left[0, \frac{1}{\sqrt{3}}\right]$ and $\|x\| \leq \sqrt{1-3 t^{2}}$, in particular $A$ is bounded.

(b) Every element $x \oplus t \in X$ satisfying $t \in\left[0, \frac{1}{\sqrt{3}}\right]$ and $\|x\| \leq \sqrt{\frac{1-3 t^{2}}{1+t^{2}}}$ belongs to $A$.

(c) A is closed.

(d) $A$ is convex.

(e) $A$ is $S C D$.

Proof. Conditions (a) and (b) follow immediately from (2.2) and (2.1). (c) follows from the continuity of the map $x \oplus t \mapsto\|x\|_{t}$. To check (d), that $A$ is convex, note that the set can be rewritten as

$$
A=\{x \oplus t \in X: H(\|x\|, \varphi(x), t) \leq 1\} \cap\{x \oplus t \in X: t \geq 0\}
$$

where $H(r, s, t):=r^{2}+t^{2} s^{2}+3 t^{2}$. $H$ is a convex function on $[0,1]^{3}$, indeed its Hessian matrix

$$
\left(\begin{array}{ccc}
2 & 0 & 0 \\
0 & 2 t^{2} & 4 t s \\
0 & 4 t s & 6+2 s^{2}
\end{array}\right)
$$

is positive definite on $(0,1)^{3}$, since the determinants of its principal minors are all positive on this domain: $\Delta_{1}=2, \Delta_{2}=4 t^{2}$ and $\Delta_{3}=12 t^{2}\left(1-s^{2}\right)$. Furthermore, $H$ is nondecreasing in each variable when considered defined on $[0,1]^{3}$, so for $x_{i} \oplus t_{i} \in A(i=1,2)$ and $0 \leq \lambda \leq 1$ we have that

$$
\begin{aligned}
& H\left(\left\|\lambda x_{1}+(1-\lambda) x_{2}\right\|, \varphi\left(\lambda x_{1}+(1-\lambda) x_{2}\right), \lambda t_{1}+(1-\lambda) t_{2}\right) \\
& \leq H\left(\lambda\left\|x_{1}\right\|+(1-\lambda)\left\|x_{2}\right\|, \lambda \varphi\left(x_{1}\right)+(1-\lambda) \varphi\left(x_{2}\right), \lambda t_{1}+(1-\lambda) t_{2}\right) \\
& \leq \lambda H\left(\left\|x_{1}\right\|, \varphi\left(x_{1}\right), t_{1}\right)+(1-\lambda) H\left(\left\|x_{2}\right\|, \varphi\left(x_{2}\right), t_{2}\right) \\
& \leq 1-\lambda+\lambda=1 .
\end{aligned}
$$

Therefore $\lambda\left(x_{1} \oplus t_{1}\right)+(1-\lambda)\left(x_{2} \oplus t_{2}\right) \in A$. 
We finally prove (e), that $A$ is an SCD set, by showing that it is huskable (see Proposition 1.4(ii)). To this end, denote $\tilde{A}=\{x \oplus t \in A: 0<t<1 / \sqrt{3}$, $\left.\|x\|_{t}^{2}=1-3 t^{2}\right\}$. Evidently, $\overline{\operatorname{conv}}(\tilde{A})=A$, so it remains to demonstrate the following statement:

For every $\varepsilon>0$ and every $x_{0} \oplus t_{0} \in \tilde{A}$ there is a relatively weakly open subset of $A$ containing $x_{0} \oplus t_{0}$ with $\|\cdot\|_{\infty^{-}}$ diameter less than $4 \varepsilon$.

For this, let us write briefly $r_{0}:=\left(1-3 t_{0}^{2}\right)^{1 / 2}=\left\|x_{0}\right\|_{t_{0}}$.

Since $x_{0}$ is a strongly exposed point of $r_{0} B_{\left(E,\|\cdot\|_{t_{0}}\right)}$, there exist $x_{0}^{*} \in$ $S_{\left(E^{*},\|\cdot\|_{t_{0}}^{*}\right)}$ and $\beta_{0} \in(0,1)$ satisfying:

(i) $x_{0}^{*}\left(x_{0}\right)=r_{0}$.

(ii) If $x \in r_{0} B_{\left(E,\|\cdot\|_{0}\right)}$ and $x_{0}^{*}(x)>\beta_{0} r_{0}$, then $\left\|x-x_{0}\right\|<\varepsilon$.

Take $\delta>0$ small enough so that

$$
x_{0}^{*}\left(x_{0}\right)>\beta_{0}\left(r_{0}+2 \delta\right) \text { and } \frac{2 \delta}{2 \delta+r_{0}}+\frac{\delta^{2}}{2}<\varepsilon .
$$

Consider the relatively weakly open subset $W$ of $A$ given by

$$
W:=\left\{x \oplus t \in A: x_{0}^{*}(x)>\beta_{0}\left(r_{0}+2 \delta\right),\left|t-t_{0}\right|<\delta^{2} / 2\right\} .
$$

It is immediate that $x_{0} \oplus t_{0} \in W$. Furthermore, given $x \oplus t \in W$ we have that $\left|t^{2}-t_{0}^{2}\right|<\delta^{2}$ and hence

$$
\begin{aligned}
\|x\|_{t_{0}} & =\left(\|x\|^{2}+t_{0}^{2} \varphi(x)\right)^{1 / 2} \\
& =\left(\|x\|_{t}^{2}+\left(t_{0}^{2}-t^{2}\right) \varphi(x)\right)^{1 / 2} \\
& \leq\left(\|x\|_{t}^{2}+\left|t_{0}^{2}-t^{2}\right|\right)^{1 / 2} \\
& \leq\left(1-3 t^{2}+\left|t_{0}^{2}-t^{2}\right|\right)^{1 / 2} \\
& \leq\left(1-3 t_{0}^{2}+4 \delta^{2}\right)^{1 / 2} \\
& \leq r_{0}+2 \delta .
\end{aligned}
$$

The last inequality together with (2.3) gives that

$$
\left\|\frac{r_{0} x}{r_{0}+2 \delta}\right\|_{t_{0}} \leq r_{0} \quad \text { and } \quad x_{0}^{*}\left(\frac{r_{0} x}{r_{0}+2 \delta}\right)>\beta_{0} r_{0} .
$$

By (ii) it follows that

$$
\varepsilon>\left\|\frac{r_{0} x}{r_{0}+2 \delta}-x_{0}\right\| \geq\left\|x-x_{0}\right\|-\|x\| \frac{2 \delta}{r_{0}+2 \delta} \geq\left\|x-x_{0}\right\|-\frac{2 \delta}{r_{0}+2 \delta},
$$

and therefore

$\left\|x \oplus t-x_{0} \oplus t_{0}\right\|_{\infty}=\max \left\{\left\|x-x_{0}\right\|,\left|t-t_{0}\right|\right\}<\max \left\{\varepsilon+\frac{2 \delta}{2 \delta+r_{0}}, \frac{\delta^{2}}{2}\right\}<2 \varepsilon$.

We conclude then that the diameter of $W$ is less than $4 \varepsilon$ finishing the proof of the statement above.

Remark also that $A$ in the above Proposition has two more evident properties: it has non-empty interior, and for every $x \oplus t \in A$ also $(-x) \oplus t \in A$. 
Theorem 2.2. In every Banach space $X$ with the Daugavet property there are convex closed bounded $S C D$ sets $A, D \subset X$ whose intersection $A \cap D$ is not $S C D$.

Proof. Let $A$ and $E$ be as in Proposition 2.1, and let $D=-A$. Both sets are SCD by Proposition 2.1 although $A \cap D=B_{E}$ is not.

2.2. Sum and union of SCD sets. For $B_{1}, B_{2} \subset X$ we denote, as usual, by $B_{1}+B_{2}$ the corresponding Minkowski sum: $B_{1}+B_{2}=\left\{b_{1}+b_{2}: b_{1} \in\right.$ $\left.B_{1}, b_{2} \in B_{2}\right\}$. The need to consider various Minkowski sums appears in many instances, in particular in the applications of SCD sets to operator theory. The first theorem of that kind appeared in [2, Corollary 3.9], where inheritance of the property SCD of two spaces by their direct sum was demonstrated. The next step (that has important applications) was done in [10, Theorem 2.1]: the direct sum of two hereditarily SCD sets is a hereditarily SCD set again (hereditarily SCD means that all subsets are $\mathrm{SCD})$. In the same paper it was demonstrated that in the statement of the latter result the direct sum cannot be substituted by the Minkowski sum. Namely, in [10, Corollary 2.2] it is demonstrated that the Minkowski sum of two hereditarily SCD sets need not be hereditarily SCD. Unfortunately, the statement of [10, Corollary 2.2] as it appeared in the paper, viz. "The sum of two hereditarily SCD sets need not be an SCD set," contains a misleading misprint: the second word "hereditarily" is missing. The construction in [10, Corollary 2.2] consists of two separable RNP subsets $U, V \subset \ell_{1} \oplus_{\infty}$ $C[0,1]$ such that $U+V \supset B_{C[0,1]}$ which makes $U+V$ not hereditarily SCD. Nevertheless, [14, comments after Prop. 1.7], $\overline{U+V}$ is the closed convex hull of its strongly exposed points, so $U+V$ is SCD. The authors noted that painful misprint only some years after the publication (see Editor's comment to Zentralblatt review Zbl 1210.46010), and since then it has remained an open question whether the statement with the misprint is incidentally also correct. In this subsection we answer a related question, demonstrating that the Minkowski sum of two SCD sets need not be SCD. We also give an analogous result about unions of SCD sets.

At first, remark the following easy properties:

Lemma 2.3. Let $B_{1}, B_{2} \subset X$ be non-empty bounded sets and let $x^{*} \in X^{*}$, $\varepsilon>0$. We then have the following properties:

(i) $S\left(B_{1}, x^{*}, \varepsilon / 2\right)+S\left(B_{2}, x^{*}, \varepsilon / 2\right) \subset S\left(B_{1}+B_{2}, x^{*}, \varepsilon\right)$.

(ii) If $a \in B_{1}, b \in B$ satisfy that $a+b \in S\left(B_{1}+B_{2}, x^{*}, \varepsilon\right)$, then $a \in$ $S\left(B_{1}, x^{*}, \varepsilon\right)$ and $b \in S\left(B_{2}, x^{*}, \varepsilon\right)$.

The above Lemma and Lemma 1.2 imply the following result.

Lemma 2.4. Let $B_{1}, B_{2} \neq \emptyset$ be bounded subsets of a Banach space $X$. Then the following assertions are equivalent:

(a) $B_{1}+B_{2}$ is $S C D$.

(b) There exists a countable family $\left(x_{n}^{*}, \varepsilon_{n}\right) \in X^{*} \times(0,+\infty)$ satisfying that for every $\left(x^{*}, \varepsilon\right) \in X^{*} \times(0,+\infty)$ there is an $m \in \mathbb{N}$ such that

$S\left(B_{1}, x_{m}^{*}, \varepsilon_{m}\right) \subset S\left(B_{1}, x^{*}, \varepsilon\right) \quad$ and $\quad S\left(B_{2}, x_{m}^{*}, \varepsilon_{m}\right) \subset S\left(B_{2}, x^{*}, \varepsilon\right)$. 
Proof. (a) $\Rightarrow$ (b): Let $S_{n}=S\left(B_{1}+B_{2}, x_{n}^{*}, 2 \varepsilon_{n}\right)$ with $\left(x_{n}^{*}, \varepsilon_{n}\right) \in X^{*} \times(0,+\infty)$, $n \in \mathbb{N}$, be slices of $B_{1}+B_{2}$ which form a determining sequence. Let us demonstrate that $\left(x_{n}^{*}, \varepsilon_{n}\right)$ form the sequence we need for (b). Indeed, according to Lemma 1.2 for every $\left(x^{*}, \varepsilon\right) \in X^{*} \times(0,+\infty)$ there is $m \in \mathbb{N}$ such that $S_{m} \subset S\left(B_{1}+B_{2}, x^{*}, \varepsilon\right)$, and by (i) of Lemma 2.3 also $S\left(B_{1}, x_{m}^{*}, \varepsilon_{m}\right)+$ $S\left(B_{2}, x_{m}^{*}, \varepsilon_{m}\right) \subset S\left(B_{1}+B_{2}, x^{*}, \varepsilon\right)$. An application of (ii) of Lemma 2.3 gives us the desired inclusions (2.4).

(b) $\Rightarrow$ (a): Assume $\left(x_{n}^{*}, \varepsilon_{n}\right) \in X^{*} \times(0,+\infty)$ are from (b), and let us demonstrate that the slices $S_{n}=S\left(B_{1}+B_{2}, x_{n}^{*}, \varepsilon_{n}\right)$ form a determining sequence of slices for $B_{1}+B_{2}$. Fix a slice $S\left(B_{1}+B_{2}, x^{*}, 2 \varepsilon\right)$ with $x^{*} \in X^{*} \backslash\{0\}$, $\varepsilon>0$ and, using (b), select an $m$ for which (2.4) takes place. We are going to demonstrate that $S\left(B_{1}+B_{2}, x_{m}^{*}, \varepsilon_{m}\right) \subset S\left(B_{1}+B_{2}, x^{*}, 2 \varepsilon\right)$. Indeed, let $x \in S\left(B_{1}+B_{2}, x_{m}^{*}, \varepsilon_{m}\right)$ be an arbitrary element. Then it is of the form $x=a+b, a \in B_{1}, b \in B_{2}$, and, by (ii) of Lemma 2.3, $a \in S\left(B_{1}, x_{m}^{*}, \varepsilon_{m}\right)$, $b \in S\left(B_{2}, x_{m}^{*}, \varepsilon_{m}\right)$. It remains to apply (i) of Lemma 2.3:

$$
\begin{aligned}
x=a+b & \subset S\left(B_{1}, x_{m}^{*}, \varepsilon_{m}\right)+S\left(B_{2}, x_{m}^{*}, \varepsilon_{m}\right) \\
& \subset S\left(B_{1}, x^{*}, \varepsilon\right)+S\left(B_{2}, x^{*}, \varepsilon\right) \subset S\left(B_{1}+B_{2}, x^{*}, 2 \varepsilon\right) .
\end{aligned}
$$

The above lemma leads to the following result.

Theorem 2.5. Let $B_{1}, B_{2}$ be non-empty bounded subsets of a Banach space $X$ such that $B_{1}+B_{2}$ is $S C D$. Then, $B_{1}$ (and so also $B_{2}$ ) is $S C D$.

Proof. Let $\left(x_{n}^{*}, \varepsilon_{n}\right) \in X^{*} \times(0,+\infty)$ be the family from (b) of Lemma 2.4, then the slices $S\left(B_{1}, x_{m}^{*}, \varepsilon_{m}\right)$ form a determining sequence for $B_{1}$.

The next proposition explains some difficulties that arise when one has to demonstrate that a non-convex set is SCD.

Proposition 2.6. There are non-convex non-SCD sets containing a determining sequence of relatively weakly open subsets. Such examples exist in every Banach space with the Daugavet property.

Proof. Let $X$ be a space with the Daugavet property (as before it can be assumed separable), $E$ be a 1-codimensional closed subspace. Then $X$ is isomorphic to $E \oplus_{\infty} \mathbb{R}$. Take a sequence $\left(x_{n}\right)$ in the unit ball of $E$ such that both subsequences $\left(x_{2 n}\right)_{n \in \mathbb{N}}$ and $\left(x_{2 n-1}\right)_{n \in \mathbb{N}}$ are dense and a sequence of $t_{n} \in(0,1)$ such that $t_{2 n} \rightarrow 0$ and $t_{2 n+1} \rightarrow 1$. The set in question will be the following subset of $E \oplus_{\infty} \mathbb{R}$ :

$$
U=\left\{x_{n} \oplus t_{n}: n \in \mathbb{N}\right\} .
$$

This set is quickly seen to be discrete in the weak topology, so $\left\{\left\{x_{n} \oplus t_{n}\right\}: n \in \mathbb{N}\right\}$ is the requested determining sequence of relatively weakly open subsets. On the other hand the closed convex hull of $U$ equals $B_{E} \oplus[0,1]$ which, according to Theorem 2.5, is not SCD because the unit ball of $E$ is not SCD.

Now we are ready for the first main result of the subsection demonstrating that the converse to Theorem 2.5 is not true.

Theorem 2.7. In every Banach space $X$ with the Daugavet property there are convex closed bounded $S C D$ sets $A, D \subset X$ whose sum $A+D$ is not $S C D$. 
Proof. We will use the same sets $A, D \subset X$ as in Theorem 2.2:

$$
\begin{aligned}
& A=\left\{x \oplus t:\|x\|_{t} \leq 1-3 t^{2}, t \geq 0\right\}, \\
& D=-A=\left\{x \oplus t:\|x\|_{t} \leq 1-3 t^{2}, t \leq 0\right\},
\end{aligned}
$$

whose intersection is $B_{E}$. It has already been shown in Proposition 2.1 that $A$ and $D$ are SCD.

To see that the sum $A+D$ is not SCD we will argue by contradiction. If we assume that $A+D$ is SCD then we could find a countable family $\left(x_{n}^{*}, \varepsilon_{n}\right) \in S_{X^{*}} \times(0,1)$ as in Lemma 2.4. Notice that we can write $x_{n}^{*}=$ $y_{n}^{*} \oplus \lambda_{n} \in X^{*}=E^{*} \oplus \mathbb{R}$. Since $B_{E}$ is not SCD we can find $y^{*} \in S_{E^{*}}$ and $\delta \in(0,1)$ such that for every $n \in \mathbb{N}$

$$
S\left(B_{E}, y_{n}^{*}, \varepsilon_{n}\right) \not \subset S\left(B_{E}, y^{*}, \delta\right) .
$$

Considering the element $x^{*}=y^{*} \oplus 0 \in B_{X^{*}}$ we have that there is $k \in \mathbb{N}$ satisfying

$$
S\left(A, x_{k}^{*}, \varepsilon_{k}\right) \subset S\left(A, x^{*}, \delta\right) \quad \text { and } \quad S\left(D, x_{k}^{*}, \varepsilon_{k}\right) \subset S\left(D, x^{*}, \delta\right)
$$

from which it easily follows that

$$
S\left(A, x_{k}^{*}, \varepsilon_{k}\right) \cup S\left(D, x_{k}^{*}, \varepsilon_{k}\right) \subset\left\{x \oplus t \in X: x \in S\left(B_{E}, y^{*}, \delta\right)\right\} .
$$

We now claim that

$$
S\left(B_{E}, y_{k}^{*}, \varepsilon_{k}\right) \subset S\left(A, x_{k}^{*}, \varepsilon_{k}\right) \cup S\left(D, x_{k}^{*}, \varepsilon_{k}\right)
$$

which together with (2.6) leads to

$$
S\left(B_{E}, y_{k}^{*}, \varepsilon_{k}\right) \subset S\left(B_{E}, y^{*}, \delta\right),
$$

contradicting (2.5) and finishing the proof. To show the validity of the claim we distinguish two cases. Assuming that $\lambda_{k} \leq 0$ we get that $\sup \left\{x_{k}^{*}(a)\right.$ : $a \in A\}=\sup \left\{y_{k}^{*}(x): x \in B_{E}\right\}=\left\|y_{k}^{*}\right\|$. As a consequence, $S\left(B_{E}, y_{k}^{*}, \varepsilon_{k}\right) \subset$ $S\left(A, x_{k}^{*}, \varepsilon_{k}\right)$. On the other hand, if $\lambda_{k} \geq 0$ then analogously $\sup \left\{x_{k}^{*}(d): d \in\right.$ $D\}=\sup \left\{y_{k}^{*}(x): x \in B_{E}\right\}$ and therefore $S\left(B_{E}, y_{k}^{*}, \varepsilon_{k}\right) \subset S\left(D, x_{k}^{*}, \varepsilon_{k}\right)$.

Nevertheless, for the direct sum of SCD sets the situation remains simple (for hereditarily SCD sets that was proved earlier in [10, Theorem 2.1]).

Theorem 2.8. Let $B_{1} \subset X_{1}, B_{2} \subset X_{2}$ be bounded subsets of a Banach space $X=X_{1} \oplus X_{2}$, and suppose that $B_{1}, B_{2}$ are $S C D$. Then $B_{1}+B_{2}$ is $S C D$.

Proof. Let $S\left(B_{i}, y_{n, i}^{*}, \delta_{n, i}\right), y_{n, i}^{*} \in X_{i}^{*}, n \in \mathbb{N}$, form determining sequences of slices for $B_{i}, i=1,2$. Then, the collection of functionals $x_{n, m}^{*}=y_{n, 1}^{*} \oplus y_{m, 2}^{*}$ and corresponding $\varepsilon_{n, m}=\min \left\{\delta_{n, 1}, \delta_{m, 2}\right\}$ will be a countable family that satisfies condition (b) of Lemma 2.4.

And now for the last of the promised main examples of the subsection.

Theorem 2.9. In every Banach space $X$ with the Daugavet property there is an $S C D$ set $B$ such that $B \cup(-B)$ is not $S C D$.

Proof. We follow the notation of Proposition 2.1. Let $\alpha:=1 /(2 \sqrt{3})$ and $B:=A-\alpha e_{0}=\{x \oplus(t-\alpha): x \oplus t \in A\}$, where $A$ is the set in (2.2). We claim that

$$
\operatorname{conv}(B \cup(-B))=B_{E} \oplus[-\alpha, \alpha] .
$$


Indeed, it is clear that $B$ is contained in $B_{E} \oplus[-\alpha, \alpha]$, and so is $-B$. For the converse, use simply that $B_{E}-\alpha e_{0} \subset B, B_{E}+\alpha e_{0} \subset-B$, and consequently

$$
B_{E} \oplus[-\alpha, \alpha]=\operatorname{conv}\left(B_{E}-\alpha e_{0}\right) \cup\left(B_{E}+\alpha e_{0}\right) \subset \operatorname{conv}(B \cup(-B)) .
$$

Finally, if $B \cup(-B)$ were SCD, then $B_{E} \oplus[-\alpha, \alpha]$ would be SCD by Lemma 1.3. But it was already remarked above that this is never the case by Theorem 2.5, as $B_{E}$ is not SCD because of the Daugavet property of $E$ [2, Example 2.13].

\section{Symmetrization of the EXAmples}

In the most important applications of SCD sets, the sets which appear are balls and images of balls under the action of linear operators. So, it would be natural to ask whether examples demonstrating non-stability of the property SCD can be constructed to be balls of some equivalent norms, that is, to be convex closed bounded symmetric bodies. The keyword here is "symmetric" because the examples that we have constructed above possess all the remaining properties of being convex closed bounded, and to have nonempty interior. In this section we apply a natural symmetrization procedure which helps to obtain symmetric examples from non-symmetric ones.

Let $U$ be a bounded non-empty subset of a Banach space $X$. By symmetrization of $U$ we will mean the following subset of $\operatorname{Sym}(U) \subset X \oplus_{\infty} \mathbb{R}$ :

$$
\operatorname{Sym}(U)=\operatorname{aconv}(U \oplus 1) .
$$

Lemma 3.1. Let $U, V \neq \emptyset$ be bounded subsets such that $U$ is contained in a closed hyperplane $H_{0}$, and $V$ lies on one side of $H_{0}$ at a positive distance from $H_{0}$. Then, every slice of $U$ is at the same time a slice of $U \cup V$.

Proof. Without loss of generality we can assume that $0 \in U$, and that $U, V \subset$ $B_{X}$ (this can be done by shifting and scaling). Then there are an $x_{0}^{*} \in S_{X^{*}}$ and $\varepsilon_{0}>0$ such that $H_{0}=\operatorname{ker} x_{0}^{*} \supset U$, and $V \subset\left\{x \in X: x_{0}^{*}(x)<-\varepsilon_{0}\right\}$. Let $x^{*} \in S_{X^{*}}$ and let $S=S\left(U, x^{*}, \varepsilon\right)$ be a slice of $U$. Denote $r=\sup _{x \in U} x^{*}(x) \in$ $[-1,1]$ and consider for every $t>0$ the functional $x_{t}^{*}=x^{*}+t x_{0}^{*}$. Since on $U$ the values of $x_{t}^{*}$ and of $x^{*}$ are the same, $S=S\left(U, x_{t}^{*}, \varepsilon\right)$ for all $t>0$. We are going to demonstrate that for some values of $t>0$ the slice $S_{t}=$ $S\left(U \cup V, x_{t}^{*}, \varepsilon\right)$ of $U \cup V$ also equals $S$, which will complete our proof.

So our goal is to show that there is a $t>0$ such that $S_{t} \cap V=\emptyset$. Assume to the contrary that for every $t>0$ there is an element $v_{t} \in V \cap S_{t}$. Then

$$
\begin{aligned}
1-t \varepsilon_{0} & \geq x^{*}\left(v_{t}\right)+t x_{0}^{*}\left(v_{t}\right)=x_{t}^{*}\left(v_{t}\right) \\
& >\sup _{x \in U \cup V} x_{t}^{*}(x)-\varepsilon \\
& =\max \left\{\sup _{x \in U} x^{*}(x), \sup _{x \in V} x^{*}(x)+t x_{0}^{*}(x)\right\}-\varepsilon \\
& \geq \max \left\{r, r-t \varepsilon_{0}\right\}-\varepsilon=r-\varepsilon,
\end{aligned}
$$

which means that $t<\frac{1+\varepsilon-r}{\varepsilon_{0}}$. This is a contradiction.

Lemma 3.2. If under the conditions of Lemma 3.1 $U \cup V$ is $S C D$, then $U$ is also an $S C D$ set. 
Proof. Let $\left\{V_{n}: n \in \mathbb{N}\right\}$ be a determining sequence of slices of $U \cup V$. Denote $N_{1}=\left\{n \in \mathbb{N}: V_{n} \cap U \neq \emptyset\right\}$. Then $S_{n}:=V_{n} \cap U, n \in N_{1}$, are slices of $U$. We are going to demonstrate that the collection $\left\{S_{n}: n \in N_{1}\right\}$ is determining for $U$, which will do the job. Let us use Lemma 1.2. Consider a slice $S$ of $U$. Then, by Lemma 3.1, $S$ is at the same time a slice of $U \cup V$. So, there is an $n \in \mathbb{N}$ such that $V_{n} \subset S$, but this $n$ automatically belongs to $N_{1}$.

Lemma 3.3. The following conditions for a bounded non-empty subset $U \subset$ $X$ are equivalent:

(i) $U$ is $S C D$,

(ii) $(U \oplus 1) \cup-(U \oplus 1)$ is $S C D$,

(iii) $\operatorname{Sym}(U)$ is $S C D$.

Proof. Taking into account that $\operatorname{Sym}(U)=\operatorname{conv}((U \oplus 1) \cup-(U \oplus 1))$ the equivalence (ii) $\Leftrightarrow$ (iii) follows from Lemma 1.3.

(i) $\Rightarrow$ (ii): Let $\left\{S_{n}: n \in \mathbb{N}\right\}$ be a determining sequence of slices of $U$. Then, the $V_{n}:=S_{n} \oplus 1$ form a determining sequence of slices of $U \oplus 1$ and the $-V_{n}$ form a determining sequence of slices of $-(U \oplus 1)$. By Lemma 3.1, $\pm V_{n}$ are also slices of $(U \oplus 1) \cup-(U \oplus 1)$. But then the countable collection $\left\{ \pm V_{n}: n \in \mathbb{N}\right\}$ forms a determining sequence of slices of $(U \oplus 1) \cup-(U \oplus 1)$. Indeed, let $V \subset X \oplus_{\infty} \mathbb{R}$ intersect all $\pm V_{n}, n \in \mathbb{N}$. Then, since $\left\{V_{n}: n \in \mathbb{N}\right\}$ is determining for $U \oplus 1$, we have $\overline{\operatorname{conv}}(V) \supset U \oplus 1$ and since the $-V_{n}$ form a determining sequence of slices of $-(U \oplus 1)$ we also have $\overline{\operatorname{conv}}(V) \supset-(U \oplus 1)$, which completes the proof of the implication (i) $\Rightarrow$ (ii).

(ii) $\Rightarrow$ (i): Applying Lemma 3.2 we obtain that $U \oplus 1$ is $\mathrm{SCD}$, but $U \oplus 1$ is a shift of $U$, so $U$ is also SCD.

The next example is based on the elementary fact that the convex hull of the union of symmetrized sets $\operatorname{Sym}\left(U_{1}\right) \cup \operatorname{Sym}\left(U_{2}\right)$ is equal to the symmetrized union $\operatorname{Sym}\left(U_{1} \cup U_{2}\right)$ :

$$
\operatorname{conv}\left(\operatorname{Sym}\left(U_{1}\right) \cup \operatorname{Sym}\left(U_{2}\right)\right)=\operatorname{Sym}\left(U_{1} \cup U_{2}\right) .
$$

Indeed, the left hand side is convex and symmetric, contains $\left(U_{1} \cup U_{2}\right) \oplus 1$, so contains $\operatorname{Sym}\left(U_{1} \cup U_{2}\right)$. Conversely, the right hand side is convex, contains $\operatorname{Sym}\left(U_{1}\right)$ and $\operatorname{Sym}\left(U_{2}\right)$, so contains the convex hull conv $\left(\operatorname{Sym}\left(U_{1}\right) \cup \operatorname{Sym}\left(U_{2}\right)\right)$.

Theorem 3.4. In every Banach space $Y$ with the Daugavet property there are convex closed bounded symmetric sets $\tilde{B}_{1}, \tilde{B}_{2} \subset Y$ which are $S C D$ sets, but whose union $\tilde{B}_{1} \cup \tilde{B}_{2}$ is not $S C D$. If, additionally, $Y$ is separable, then these $\tilde{B}_{1}, \tilde{B}_{2} \subset Y$ can be chosen to have non-empty interior.

Proof. It is sufficient to consider the case of separable $Y$ (otherwise, substitute it by a separable subspace with the Daugavet property). Let $X$ be a onecodimensional closed subspace of $Y$. Then $X$ also has the Daugavet property. Our $Y$ is isomorphic to $X \oplus_{\infty} \mathbb{R}$, so it is sufficient to construct the requested example in $X \oplus_{\infty} \mathbb{R}$. Let $B$ and $-B$ be SCD sets from Theorem 2.9, and take $B_{1}=\operatorname{Sym}(B), B_{2}=\operatorname{Sym}(-B)$. Since $B,-B$ are convex, bounded and have non-empty interior, $B_{1}$ and $B_{2}$ are convex bounded symmetric bodies which are SCD by the previous Lemma 3.3. Also, $\operatorname{conv}\left(B_{1} \cup B_{2}\right)=\operatorname{Sym}(B \cup(-B))$, so by the same Lemma $3.3 \operatorname{conv}\left(B_{1} \cup B_{2}\right)$ is not SCD, and consequently $B_{1} \cup B_{2}$ is not SCD. To finish the proof define $\tilde{B}_{1}$ and $\tilde{B}_{2}$ to be the closures of $B_{1}$ and $B_{2}$ and apply Lemma 1.3. 
In order to proceed with the symmetrization of the example about the sum of SCD sets, we first need a natural lemma.

Lemma 3.5. Let $U_{1}, U_{2} \subset X$ be bounded not empty subsets. Then,

$$
\overline{\operatorname{conv}}\left(U_{1}+U_{2}\right)=\overline{\operatorname{conv}\left(U_{1}\right)+\operatorname{conv}\left(U_{2}\right)} .
$$

Proof. Both the right hand side and the left hand side of the equality in question are convex closed sets, so each of them is the intersection of all half-spaces that contain it. In other words, in order to prove the equality it is sufficient to demonstrate that for every $x^{*} \in X^{*}$

$$
\sup x^{*}\left(\overline{\operatorname{conv}}\left(U_{1}+U_{2}\right)\right)=\sup x^{*}\left(\overline{\operatorname{conv}\left(U_{1}\right)+\operatorname{conv}\left(U_{2}\right)}\right) .
$$

This equality is easily seen to be true, because its right hand side and left hand side are both equal to $\sup x^{*}\left(U_{1}\right)+\sup x^{*}\left(U_{2}\right)$.

Theorem 3.6. In every Banach space $Y$ with the Daugavet property there are convex closed bounded symmetric $S C D$ sets (which in the separable case can be chosen to be bodies) $C_{1}, C_{2} \subset Y$ whose sum $C_{1}+C_{2}$ is not $S C D$.

Proof. As before, we can reduce the situation to a separable space of the form $X \oplus_{\infty} \mathbb{R}$, where $X$ has the Daugavet property. Let $B_{1}:=A, B_{2}:=D$ be SCD subsets of $X$ from Theorem 2.7 such that $B_{1}+B_{2}$ is not SCD, and take $C_{1}=\overline{\operatorname{Sym}\left(B_{1}\right)}, C_{2}=\overline{\operatorname{Sym}\left(B_{2}\right)}$, which are closed convex bounded symmetric SCD bodies. It remains to demonstrate that $C_{1}+C_{2}$ is not SCD. Using Lemma 3.5 we can see that

$$
\begin{aligned}
\overline{C_{1}+C_{2}} & =\overline{\operatorname{conv}\left(\left(B_{1} \oplus 1\right) \cup-\left(B_{1} \oplus 1\right)\right)+\operatorname{conv}\left(\left(B_{2} \oplus 1\right) \cup-\left(B_{2} \oplus 1\right)\right)} \\
& =\overline{\operatorname{conv}}\left(\left(\left(B_{1} \oplus 1\right) \cup-\left(B_{1} \oplus 1\right)\right)+\left(\left(B_{2} \oplus 1\right) \cup-\left(B_{2} \oplus 1\right)\right)\right) .
\end{aligned}
$$

According to Lemma 1.3, it is sufficient to show that the set

$$
\begin{aligned}
\left(\left(B_{1} \oplus 1\right) \cup-\left(B_{1} \oplus 1\right)\right) & +\left(\left(B_{2} \oplus 1\right) \cup-\left(B_{2} \oplus 1\right)\right) \\
=\left(\left(B_{1}+B_{2}\right) \oplus 2\right) & \cup\left(\left(B_{1}-B_{2}\right) \oplus 0\right) \\
& \cup\left(\left(B_{2}-B_{1}\right) \oplus 0\right) \cup\left(-\left(B_{1}+B_{2}\right) \oplus(-2)\right)
\end{aligned}
$$

is not SCD. With the help of Lemma 3.1 this can be deduced from the fact that $B_{1}+B_{2}$ is not SCD exactly the same way as the implication (ii) $\Rightarrow$ (i) of Lemma 3.3, because $\left(B_{1}+B_{2}\right) \oplus 2$ lies in the hyperplane of those elements whose second coordinate equals 2 , and the rest of the set lies at a distance at least 2 from that hyperplane.

Before coming to the symmetrization of the non-SCD intersection example, one more easy remark.

Lemma 3.7. Let $U_{0}, U_{1} \subset X$ be non-empty subsets with $U_{0} \subset U_{1}$, and let $U_{1}$ be convex. Then, $U_{\lambda}:=\lambda U_{1}+(1-\lambda) U_{0}$ increases when $\lambda \in[0,1]$ increases.

Proof. Let $0 \leq \lambda \leq \mu \leq 1$. Then

$$
\begin{aligned}
U_{\mu}=\mu U_{1}+(1-\mu) U_{0} & =\lambda U_{1}+(\mu-\lambda) U_{1}+(1-\mu) U_{0} \\
& \supset \lambda U_{1}+(\mu-\lambda) U_{0}+(1-\mu) U_{0} \\
& \supset \lambda U_{1}+(1-\lambda) U_{0}=U_{\lambda} .
\end{aligned}
$$


Also remark that if $U \subset X$ is convex, then $\operatorname{Sym}(U) \subset X \oplus_{\infty} \mathbb{R}$ can be written as

$$
\operatorname{Sym}(U)=\{(t u-(1-t) v) \oplus(2 t-1): u, v \in U, t \in[0,1]\} .
$$

In other words,

$$
\operatorname{Sym}(U)=\bigcup_{t \in[0,1]}(t U-(1-t) U) \oplus(2 t-1) .
$$

This implies the following formula for the intersection of $\operatorname{Sym}\left(U_{1}\right) \cap \operatorname{Sym}\left(U_{2}\right)$ in the case of convex $U_{1}, U_{2} \subset X$ :

$\operatorname{Sym}\left(U_{1}\right) \cap \operatorname{Sym}\left(U_{2}\right)=\bigcup_{t \in[0,1]}\left(\left(t U_{1}-(1-t) U_{1}\right) \cap\left(t U_{2}-(1-t) U_{2}\right)\right) \oplus(2 t-1)$.

Theorem 3.8. In every (separable) Banach space $Y$ with the Daugavet property there are convex closed bounded symmetric sets (bodies) which are SCD sets, but whose intersection is not SCD.

Proof. Again, it is sufficient to consider a separable space of the form $X \oplus_{\infty}$ $\mathbb{R}$, where $X$ has the Daugavet property. Let $A \subset X$ be as in Proposition 2.1. Denote $U_{1}=A, U_{2}=-A$. We are going to demonstrate that $\operatorname{Sym}\left(U_{1}\right), \operatorname{Sym}\left(U_{2}\right) \subset X \oplus_{\infty} \mathbb{R}$ are the requested non-empty bounded convex symmetric SCD bodies such that $W:=\operatorname{Sym}\left(U_{1}\right) \cap \operatorname{Sym}\left(U_{2}\right)$ is not SCD.

Each element of $X$ is of the form $e+t e_{0}, e \in E, t \in \mathbb{R}$, and in order to avoid misunderstanding we will not use the expression $e \oplus t$ for $e+t e_{0}$ in the current proof. The notation $x \oplus t$ is reserved for elements of $X \oplus_{\infty} \mathbb{R}$, and $x^{*} \oplus \tau$ for elements of $\left(X \oplus_{\infty} \mathbb{R}\right)^{*}=X^{*} \oplus_{1} \mathbb{R}$.

For every $t \in[0,1]$ denote $A_{t}=(t A-(1-t) A) \cap((1-t) A-t A)$. Then,

$$
W=\bigcup_{t \in[0,1]}\left(A_{t} \oplus(2 t-1)\right) .
$$

Geometrically this means that the lowest level section (with $t=0$ ) of $W$ is the set $(A \cap-A) \oplus(-1)=B_{E} \oplus(-1)$, when we move to higher levels the section transforms up to $\frac{A-A}{2} \oplus 0$ when $t=\frac{1}{2}$, and then transforms back until $(A \cap-A) \oplus 1=B_{E} \oplus 1$ when $t=1$. The set $W$ is not only centrally symmetric with respect to zero, but also doubly mirror-symmetric in the following sense: for every $e \in E, a, b \in \mathbb{R}$, if $\left(e+a e_{0}\right) \oplus b \in W$, then $\left( \pm e \pm a e_{0}\right) \oplus( \pm b) \in W$ for all choices of \pm .

Let us assume to the contrary that $W$ is SCD. From this assumption we are going to deduce that $B_{E}$ is SCD, which will be the desired contradiction. Let $S_{n}=S\left(W, w_{n}^{*}, \varepsilon_{n}\right)$ form a determining sequence of slices of $W, w_{n}^{*}=$ $x_{n}^{*} \oplus \tau_{n}$. Denote also $e_{n}^{*} \in E^{*}$ and $s_{n} \in \mathbb{R}$ those elements that represent the corresponding $x_{n}^{*}$, i.e., $x_{n}^{*}\left(e+t e_{0}\right)=e_{n}^{*}(e)+s_{n} t$ for all $e \in E, t \in \mathbb{R}$. By the Bishop-Phelps theorem the set of functionals that attain their supremum on $W$ is norm-dense in the dual space, consequently, by a small perturbation argument, we may assume that each $w_{n}^{*}$ attains its supremum $R_{n}$ on $W$ at some point $w_{n}=x_{n} \oplus b_{n}=\left(e_{n}+a_{n} e_{0}\right) \oplus b_{n} \in W, b_{n}=2 t_{n}-1$, that is

$$
R_{n}:=\sup _{w \in W} w_{n}^{*}(w)=x_{n}^{*}\left(x_{n}\right)+\tau_{n} b_{n}=e_{n}^{*}\left(e_{n}\right)+s_{n} a_{n}+\tau_{n} b_{n} .
$$


We are going to show that $\tilde{S}_{n}=S\left(B_{E}, e_{n}^{*}, \varepsilon_{n}\right), n \in \mathbb{N}$, form a determining sequence of slices of $B_{E}$. Fix an arbitrary $e^{*} \in S_{E^{*}}$ and $\varepsilon \in(0,1)$. According to Lemma 1.2, our task is to find an $n \in \mathbb{N}$ such that $\tilde{S}_{n} \subset S\left(B_{E}, e^{*}, \varepsilon\right)$. Let us extend $e^{*}$ to the whole $X \oplus_{\infty} \mathbb{R}$ by the natural rule $e^{*}\left(\left(e+t_{1} e_{0}\right) \oplus t_{2}\right):=$ $e^{*}(e)$ and consider the corresponding slice $S\left(W, e^{*}, \frac{\varepsilon}{2}\right)$. Due to the same Lemma 1.2 there is an $m \in \mathbb{N}$ such that $S_{m} \subset S\left(W, e^{*}, \frac{\varepsilon}{2}\right)$. Remark that the corresponding $e_{m}^{*}$ is non-zero, otherwise with every point $\left(e+c e_{0}\right) \oplus d$ the slice $S_{m}$ would contain also $\left(c e_{0}\right) \oplus d$, thus contradicting the inclusion $S_{m} \subset$ $S\left(W, e^{*}, \frac{\varepsilon}{2}\right)$. Without loss of generality we may assume that $s_{m}, \tau_{m} \geq 0$ (here we use the symmetry of $W$ and of $S\left(W, e^{*}, \frac{\varepsilon}{2}\right)$ with respect to corresponding changes of signs). Then we can also assume $a_{m}, b_{m} \geq 0$ and consequently $t_{m} \geq \frac{1}{2}$.

By the definition, $x_{m}^{*}\left(x_{m}\right)=\sup x_{m}^{*}\left(A_{t_{m}}\right)$. We claim that in fact

$$
x_{m}^{*}\left(x_{m}\right)=\sup x_{m}^{*}\left(\left(1-t_{m}\right) A+t_{m} B_{E}\right)=\left(1-t_{m}\right) \sup x_{m}^{*}(A)+t_{m}\left\|e_{m}^{*}\right\| .
$$

Indeed, $A_{t_{m}}=\left(t_{m} A-\left(1-t_{m}\right) A\right) \cap\left(\left(1-t_{m}\right) A-t_{m} A\right) \subset\left(1-t_{m}\right) A-t_{m} A$, so $x_{m} \in A_{t_{m}}$ has a representation of the form $x_{m}=\left(1-t_{m}\right) y-t_{m} z$ with $y, z \in A$. Consequently,

$$
\begin{aligned}
x_{m}^{*}\left(x_{m}\right) & =\left(1-t_{m}\right) x_{m}^{*}(y)+t_{m} x_{m}^{*}(-z) \\
& \leq\left(1-t_{m}\right) \sup x_{m}^{*}(A)+t_{m} \sup x_{m}^{*}(-A) \\
& =\left(1-t_{m}\right) \sup x_{m}^{*}(A)+t_{m}\left\|e_{m}^{*}\right\|,
\end{aligned}
$$

where we used the positivity of $s_{m}$ in the last step. For the reverse inequality in (3.2) we can use the inclusion $A \supset B_{E}$, the inequality $t_{m} \geq 1-t_{m}$ and Lemma 3.7 which together give us the inclusion $\left(1-t_{m}\right) A+t_{m} B_{E} \subset$ $t_{m} A+\left(1-t_{m}\right) B_{E}$. This implies that

$$
\begin{aligned}
A_{t_{m}} & =\left(t_{m} A-\left(1-t_{m}\right) A\right) \cap\left(\left(1-t_{m}\right) A-t_{m} A\right) \\
& \supset\left(t_{m} A-\left(1-t_{m}\right) B_{E}\right) \cap\left(\left(1-t_{m}\right) A-t_{m} B_{E}\right) \\
& =\left(t_{m} A+\left(1-t_{m}\right) B_{E}\right) \cap\left(\left(1-t_{m}\right) A+t_{m} B_{E}\right) \\
& \supset\left(1-t_{m}\right) A+t_{m} B_{E},
\end{aligned}
$$

so

$$
x_{m}^{*}\left(x_{m}\right)=\sup x_{m}^{*}\left(A_{t_{m}}\right) \geq \sup x_{m}^{*}\left(\left(1-t_{m}\right) A+t_{m} B_{E}\right) .
$$

Thus, the formula (3.2) is proved. It remains to prove that $\tilde{S}_{m} \subset S\left(B_{E}, e^{*}, \varepsilon\right)$, or in other words that $\tilde{S}_{m} \backslash S\left(B_{E}, e^{*}, \varepsilon\right)=\emptyset$. Assume that this set is not empty, and pick an arbitrary $e \in \tilde{S}_{m} \backslash S\left(B_{E}, e^{*}, \varepsilon\right)$. Then $e \in B_{E}$ and $e$ satisfies simultaneously two inequalities:

$$
e_{m}^{*}(e)>\left\|e_{m}^{*}\right\|-\varepsilon_{m}, \quad \text { and } \quad e^{*}(e) \leq 1-\varepsilon .
$$

Take an arbitrary $g \in A$ with $x_{m}^{*}(g)>\sup x_{m}^{*}(A)-\varepsilon_{m}$. According to (3.3), $\left(1-t_{m}\right) g+t_{m} e \in A_{t_{m}}$, so

$$
\left(\left(1-t_{m}\right) g+t_{m} e\right) \oplus b_{m}=\left(\left(1-t_{m}\right) g+t_{m} e\right) \oplus\left(2 t_{m}-1\right) \in W .
$$

Then, the following inequality

$$
\begin{aligned}
w_{m}^{*}\left(\left(\left(1-t_{m}\right) g+t_{m} e\right) \oplus b_{m}\right) & =x_{m}^{*}\left(\left(1-t_{m}\right) g+t_{m} e\right)+\tau_{m} b_{m} \\
& >\left(1-t_{m}\right)\left(\sup x_{m}^{*}(A)-\varepsilon_{m}\right)+t_{m}\left(\left\|e_{m}^{*}\right\|-\varepsilon_{m}\right)+\tau_{m} b_{m} \\
& =x_{m}^{*}\left(x_{m}\right)+\tau_{m} b_{m}-\varepsilon_{m}=R_{m}-\varepsilon_{m}
\end{aligned}
$$


implies that $\left(\left(1-t_{m}\right) g+t_{m} e\right) \oplus b_{m} \in S_{m}$, and consequently $\left(\left(1-t_{m}\right) g+\right.$ $\left.t_{m} e\right) \oplus b_{m} \in S\left(W, e^{*}, \frac{\varepsilon}{2}\right)$. This means that

$$
\left(1-t_{m}\right) e^{*}(g)+t_{m} e^{*}(e)=e^{*}\left(\left(\left(1-t_{m}\right) g+t_{m} e\right) \oplus b_{m}\right)>1-\frac{\varepsilon}{2} .
$$

Together with the second condition from (3.4) this gives

$1-\frac{\varepsilon}{2}<\left(1-t_{m}\right) e^{*}(g)+t_{m} e^{*}(e)<\left(1-t_{m}\right)+t_{m}(1-\varepsilon)=1-t_{m} \varepsilon \leq 1-\frac{\varepsilon}{2}$.

This contradiction proves that $\tilde{S}_{m} \backslash S\left(B_{E}, e^{*}, \varepsilon\right)=\emptyset$.

\section{Relationship Between SCD And ASCD Sets}

In this section we prove two main results that answer [2, Question 7.5]. Namely, we demonstrate that the properties aSCD and SCD are not equivalent for general bounded closed convex sets, but in the most important case for the applications, namely that of balanced bounded closed convex sets, the equivalence holds true.

4.1. aSCD and SCD are not equivalent. In order to present the promised example of an aSCD set that is not SCD, we need some more preparatory work. We keep the notation from Proposition 2.1. In particular, $X$ is the direct sum of its subspace $E$ and a one-dimensional subspace span $e_{0}$ equipped with an equivalent norm in which it can be identified with $E \oplus_{\infty} \mathbb{R}$, and $E$ possesses the Daugavet property.

Lemma 4.1. Denote

$$
\begin{aligned}
& C_{1}=\left\{y \oplus t \in X: t \in\left[0, \frac{1}{\sqrt{3}}\right],\|y\| \leq \sqrt{\left.\frac{1-3 t^{2}}{1+t^{2}}\right\},}\right. \\
& C_{2}=\left\{y \oplus t \in X: t \in\left[0, \frac{1}{\sqrt{3}}\right],\|y\| \leq \sqrt{1-3 t^{2}}\right\} .
\end{aligned}
$$

Then for every subset $C \subset X$ satisfying $C_{1} \subset C \subset C_{2}$ and for every slice $S$ of $C$ we can find another slice $S^{\prime}=S\left(C, y^{*} \oplus \lambda, \varepsilon\right) \subset S$ with $\lambda>0$ and $S^{\prime} \cap B_{E}=\emptyset$.

Proof. We are going to prove two claims from which we can deduce the result easily.

Claim 1: Every slice of $C$ contains a slice of the form $S\left(C, x^{*}, \varepsilon\right)$ where $x^{*}=y^{*} \oplus \lambda$ with $\lambda>0$.

Let $S=S\left(C, x^{*}, \varepsilon\right)$ be a slice of $C$ with $x^{*}=y^{*} \oplus \lambda \neq 0$, and $\lambda \leq 0$. We are going to distinguish three cases:

(A) $\lambda=0$. Without loss of generality we can take $\left\|y^{*}\right\|=1$, and consequently $\sup \left\{x^{*}(a): a \in C\right\}=1$. In this case $S\left(C, y^{*} \oplus(\varepsilon / 2), \varepsilon / 2\right)$ satisfies that each of its elements $x \oplus t$ has the property

$$
y^{*}(x)+\frac{\varepsilon}{2} \geq y^{*}(x)+t \frac{\varepsilon}{2} \geq \sup \left\{\left\langle y^{*} \oplus \frac{\varepsilon}{2}, a\right\rangle: a \in C\right\}-\frac{\varepsilon}{2} \geq 1-\frac{\varepsilon}{2},
$$

that is $y^{*}(x) \geq 1-\varepsilon$. Therefore $S\left(C, y^{*} \oplus(\varepsilon / 2), \varepsilon / 2\right) \subset S\left(C, x^{*}, \varepsilon\right)$.

(B) $y^{*}=0, \lambda<0$. Without loss of generality we can take $\lambda=-1$. In this case

$$
S\left(C, x^{*}, \varepsilon\right)=\{x \oplus t \in C: t \in[0, \varepsilon)\} .
$$


Take an arbitrary $e^{*} \in S_{E^{*}}$. Taking into account that all elements $x \oplus t \in C$ satisfy $t \leq \sqrt{\frac{1-\|x\|^{2}}{3}}$, we obtain that for all $\delta<\varepsilon^{2}$

$$
\begin{aligned}
S\left(C, e^{*} \oplus 0, \delta\right) & =\left\{x \oplus t \in C: e^{*}(x)>1-\delta\right\} \\
& \subset\{x \oplus t \in C:\|x\|>1-\delta\} \\
& \subset S\left(C, x^{*}, \varepsilon\right),
\end{aligned}
$$

which reduces the problem to the case $(\mathrm{A})$.

(C) $y^{*} \neq 0, \lambda<0$. Again, without loss of generality we can take $\left\|y^{*}\right\|=1$, and since $\lambda$ is negative, $\sup \left\{x^{*}(a): a \in C\right\}=\left\|y^{*}\right\|=1$. Fix an $\eta>0$ small enough to have $\eta^{2}-\lambda \eta<\varepsilon$. In this case the slice $S\left(C, y^{*} \oplus 0, \eta^{2}\right)$ satisfies that each of its elements $x \oplus t$ has the property

$$
\sqrt{1-3 t^{2}} \geq y^{*}(x)>1-\eta^{2}
$$

which yields that $t<\eta$ and so

$$
\left\langle y^{*} \oplus \lambda, x \oplus t\right\rangle=y^{*}(x)+\lambda t \geq 1-\eta^{2}+\lambda \eta>1-\varepsilon .
$$

We obtain that

$$
S\left(C, y^{*} \oplus 0, \eta^{2}\right) \subset S\left(C, y^{*} \oplus \lambda, \varepsilon\right),
$$

which again reduces the problem to the case (A).

Claim 2: For every $x^{*}=y^{*} \oplus \lambda \in X^{*}$ with $\lambda>0$ there is $\varepsilon>0$ such that

$$
S\left(C, y^{*} \oplus \lambda, \varepsilon\right) \cap B_{E}=\emptyset .
$$

If $y^{*}=0$ then the slice $S\left(C, 0 \oplus \lambda, \frac{\lambda}{2 \sqrt{3}}\right)$ does not intersect $B_{E}$. It remains to consider $y^{*} \neq 0$. Without loss of generality we can assume that $\left\|y^{*}\right\|=1$. Then

$$
\begin{aligned}
\sup \left\{x^{*}(a): a \in C\right\} & \geq \sup \left\{x^{*}(a): a \in C_{1}\right\} \\
& =\sup _{t \in\left[0, \frac{1}{\sqrt{3}}\right]} \sup _{\|x\| \leq \sqrt{\frac{1-3 t^{2}}{1+t^{2}}}}\left\{y^{*}(x)+\lambda t\right\} \\
& =\sup _{t \in\left[0, \frac{1}{\sqrt{3}}\right]}\left\{\sqrt{\frac{1-3 t^{2}}{1+t^{2}}}+\lambda t\right\} .
\end{aligned}
$$

Denote $g(t)=\sqrt{\frac{1-3 t^{2}}{1+t^{2}}}+\lambda t$. It is standard to check that $g^{\prime}(0)=\lambda>0$ so the supremum of $g(t)$ on $\left[0, \frac{1}{\sqrt{3}}\right]$ is strictly greater than $g(0)=1$. We can then write this supremum as $1+\delta_{\lambda}$ for some $\delta_{\lambda}>0$. Finally, we are going to check that $S\left(C, y^{*} \oplus \lambda, \delta_{\lambda} / 2\right)$ satisfies the desired property: for each $x \in B_{E}$ we have that

$$
\left\langle y^{*} \oplus \lambda, x\right\rangle=y^{*}(x) \leq 1<1+\frac{\delta_{\lambda}}{2} \leq \sup \left\{x^{*}(a): a \in C\right\}-\frac{\delta_{\lambda}}{2} .
$$

This finishes the proof of Claim 2 and of our Lemma.

Remark that according to (a) and (b) of Proposition 2.1, the set $A$ satisfies the condition $C_{1} \subset A \subset C_{2}$ of Lemma 4.1. Since $A$ is SCD, this implies the following corollary. 
Proposition 4.2. Let $X$ and $A$ be as in Proposition 2.1. Then, we can choose a determining sequence of slices $S_{n}=S\left(A, x_{n}^{*}, \varepsilon_{n}\right)$ of $A$ such that $x_{n}^{*}=y_{n}^{*} \oplus \lambda_{n}$ with $\lambda_{n}>0$ and moreover $S_{n} \cap E=\emptyset$.

Now we are ready for the main result of the subsection that answers $[2$, Question 7.5] in the negative.

Theorem 4.3. Let $X$ be a Banach space with the Daugavet property. There is a convex and closed set $\tilde{C} \subset X$ which is aSCD but not $S C D$.

Proof. As before, we reduce the situation to the separable case, consider a 1-codimensional subspace $E \subset X$, and write the whole space as the direct sum $X=E \oplus \mathbb{R} e_{0}$. Let $A$ be the same set as before:

$$
A=\left\{x \oplus t \in X:\|x\|_{t}^{2}+3 t^{2} \leq 1, t \geq 0\right\} .
$$

Let $\alpha:=1 /(2 \sqrt{3})$. Select $\delta>0$ small enough so that $\delta B_{E} \oplus \alpha \subset A$ and denote $C=\overline{\operatorname{conv}}\left(B_{E} \cup\left(\delta B_{E} \oplus \alpha\right)\right)$. We are going to consider the set

$$
\tilde{C}:=A \cup(-C) \text {. }
$$

- $\tilde{C}$ is convex and closed: $A$ and $-C$ are convex and closed sets, and it is easy to deduce that so is $\tilde{C}$ using that $\tilde{C} \subset B_{E} \oplus \mathbb{R}$ and $A \cap(-C)=$ $B_{E}$. For the same reason,

$$
\tilde{C}=\overline{\operatorname{conv}}\left(A \cup\left(-\left(\delta B_{E} \oplus \alpha\right)\right)\right) .
$$

- $\tilde{C}$ is aSCD: We know by Proposition 2.1 that $A$ is SCD. If $\left(S_{n}\right)_{n \in \mathbb{N}}$ is a determining sequence of slices for $A$, then by Proposition 4.2 we can also assume that $S_{n}=S\left(A, y_{n}^{*} \oplus \lambda_{n}, \varepsilon_{n}\right)$ with $\lambda_{n}>0$ and $S_{n} \cap B_{E}=\emptyset$. This yields that $\left(S_{n}\right)_{n \in \mathbb{N}}$ is actually a sequence of slices of $\tilde{C}$. It moreover satisfies the condition of aSCD for $\tilde{C}$ : given $x_{n} \in S_{n}$ we have that $A \subset \overline{\operatorname{conv}}\left\{x_{n}: n \in \mathbb{N}\right\}$ by the choice of the sequence, so

$$
A \cup(-C) \subset A \cup(-A) \subset \overline{\operatorname{aconv}}\left\{x_{n}: n \in \mathbb{N}\right\} .
$$

- $\tilde{C}$ is not SCD: Assume that $\tilde{C}$ is SCD. Then from (4.1) we deduce that $A \cup\left(-\left(\delta B_{E} \oplus \alpha\right)\right)$ is SCD. A direct application of Lemma 3.2 gives us that $\delta B_{E} \oplus \alpha$ is $\mathrm{SCD}$, which is impossible, because this set is obtained from the non-SCD set $\delta B_{E}$ by a shift.

4.2. The case of balanced sets. Since both the definition of a balanced set and of an aSCD set depend on the scalar field, in this subsection we address both the cases of real and complex scalars.

Let $U \subset X$ be a convex closed bounded balanced set, $x^{*} \in X^{*}$ be a nonzero functional, $\varepsilon>0$. Denote $S^{b}\left(U, x^{*}, \varepsilon\right)$ the corresponding balanced slice of $U$ :

$$
S^{b}\left(U, x^{*}, \varepsilon\right)=\left\{x \in U:\left|x^{*}(x)\right|>\sup _{a \in U}\left|x^{*}(a)\right|-\varepsilon\right\} .
$$

Also, let us call a sequence of sets $V_{n} \subset U, n \in \mathbb{N}$, balanced determining for $U$ if for each $B \subset X$ that intersects all the $V_{n}, n \in \mathbb{N}$, it holds that $U \subset \overline{\operatorname{aconv}}(B)$.

The following proposition is completely analogous to Lemma 1.2. 
Lemma 4.4. Let $U \subset X$ be a convex closed bounded balanced set. Then, the following conditions on a sequence $\left\{V_{n}: n \in \mathbb{N}\right\}$ of non-empty subsets of $U$ are equivalent:

(i) $\left\{V_{n}: n \in \mathbb{N}\right\}$ is balanced determining for $U$.

(ii) Every balanced slice of $U$ contains one of the $V_{n}$.

Proof. (i) $\Rightarrow$ (ii). Assume that some balanced slice $S=S^{b}\left(U, x^{*}, \varepsilon\right)$ of $U$ does not contain any of the $V_{n}$. Then $B:=U \backslash S$ intersects all the $V_{n}$. But $B \subset\left\{x \in X:\left|x^{*}(x)\right| \leq \sup _{a \in U}\left|x^{*}(a)\right|-\varepsilon\right\}$, which is a convex closed balanced set. Consequently $\overline{\operatorname{aconv}} B \subset\left\{x \in X:\left|x^{*}(x)\right| \leq \sup _{a \in U}\left|x^{*}(a)\right|-\varepsilon\right\}$ which means that $\overline{\operatorname{aconv}} B \not \supset U$, and consequently $\left\{V_{n}: n \in \mathbb{N}\right\}$ is not balanced determining.

(ii) $\Rightarrow$ (i). Let $B \subset U$ intersect all the $V_{n}$. Then $B$ intersects all the balanced slices of $U$, so for every $x^{*} \in X^{*}$ we have $\sup _{u \in U}\left|x^{*}(u)\right|=$ $\sup _{b \in B}\left|x^{*}(b)\right|$. This means that $B^{o}=U^{o}$ and by the bipolar theorem $U=\overline{\operatorname{aconv}}(B)$.

Theorem 4.5. Let $U \subset X$ be a convex closed bounded balanced aSCD set, then $U$ is $S C D$.

Proof. 1. The real case. Let $V_{n} \subset U, n \in \mathbb{N}$, form a balanced determining sequence of slices for $U$, and let us demonstrate that the $\pm V_{n}, n \in \mathbb{N}$, are determining for $U$. According to Lemma 1.2 we must demonstrate that every slice $S=S\left(U, x^{*}, \varepsilon\right)$ of $U$ contains one of the $\pm V_{n}$. Consider the corresponding balanced slice $S^{b}=S^{b}\left(U, x^{*}, \varepsilon\right)=S \cup(-S)$. Due to Lemma 4.4, there is an $m \in \mathbb{N}$ such that $V_{m} \subset S^{b}$. Since $V_{m}$ is connected, it must be contained in one of connected components of $S^{b}$, that is either $V_{m} \subset S$, or $V_{m} \subset(-S)$. In the first case the job is done, and in the second case $-V_{m} \subset S$, which is also fine for us.

2. The complex case. Let $V_{n} \subset U, n \in \mathbb{N}$, form a balanced determining sequence of slices for $U$, and let $\left\{r_{m}\right\}_{m \in \mathbb{N}}$ be a dense subset of the unit circle $\mathbb{T}$ of $\mathbb{C}$. We will check now that the $r_{m} V_{n}, m, n \in \mathbb{N}$, are determining for $U$. According to Lemma 1.2 we must demonstrate that every slice $S=$ $S\left(U, \operatorname{Re} x^{*}, \varepsilon\right)$ of $U$ contains one of the $r_{m} V_{n}$. For this, let $\alpha=\sup _{u \in U}\left|x^{*}(u)\right|$ (which we assume to be $>0$ to avoid a trivial situation) and consider the balanced slice $S^{b}=S^{b}\left(U, x^{*}, \varepsilon / 2\right)$. Due to Lemma 4.4, there is an $n_{0} \in \mathbb{N}$ such that $V_{n_{0}} \subset S^{b}$. This means that

$$
\left\{x^{*}(v): v \in V_{n_{0}}\right\} \cap\{z \in \mathbb{C}:|z|<\alpha-\varepsilon\}=\emptyset,
$$

in fact, these convex subsets of $\mathbb{C}=\mathbb{R}^{2}$ have positive distance and can hence be strictly separated, by the Hahn-Banach theorem, so that there is some $r \in \mathbb{T}$ such that

$$
\inf _{v \in V_{n_{0}}} \operatorname{Re} r x^{*}(v)>\sup _{|z|<\alpha-\varepsilon} \operatorname{Re} r z
$$

We may assume by a perturbation argument that $r=r_{m_{0}}$ for some $m_{0}$, thus

$$
\operatorname{Re} x^{*}\left(r_{m_{0}} v\right)>\alpha-\varepsilon \quad \text { for all } v \in V_{n_{0}},
$$

in other words $r_{m_{0}} V_{n_{0}} \subset S$. 


\section{Open PROBlems}

In this small section we list several questions about SCD sets that we have been unable to solve. Some of these questions were mentioned explicitly in previous papers, some of them appeared implicitly, and some of them are motivated by the results of our paper.

(1) Does every separable Banach space that is not SCD possess the Daugavet property in some equivalent norm?

(2) Does there exist a pair $U_{1}, U_{2}$ of hereditarily SCD subsets of a Banach space such that $U_{1}+U_{2}$ is not SCD?

(3) Does the relative weak topology on a closed convex bounded SCD-set $U \subset X$ always have a countable $\pi$-basis?

(4) Is every space with an unconditional basis SCD?

(5) Must the union of two hereditarily SCD subsets of a Banach space be an SCD set?

Concerning the last problem remark that $\operatorname{conv}\left(U_{1} \cup U_{2}\right)$ need not be hereditarily SCD when $U_{1}, U_{2}$ are hereditarily SCD. Indeed, if $U_{1}, U_{2}$ are the hereditarily SCD sets from [10, Corollary 2.2] whose Minkowski sum is not hereditarily $\operatorname{SCD}$, then $\operatorname{conv}\left(U_{1} \cup U_{2}\right) \supset \frac{1}{2}\left(U_{1}+U_{2}\right)$, so $\operatorname{conv}\left(U_{1} \cup U_{2}\right)$ is not hereditarily SCD either.

\section{REFERENCES}

[1] A. Avilés, V. Kadets, M. Martín, J. Merí, V. Shepelska. Slicely countably determined Banach spaces. C. R., Math., Acad. Sci. Paris 347, no. 21-22, 1277-1280 (2009).

[2] A. Avilés, V. Kadets, M. Martín, J. Merí, V. Shepelska. Slicely countably determined Banach spaces. Trans. Amer. Math. Soc. 362, no. 9, 4871-4900 (2010).

[3] T. V. Bosenko. Strong Daugavet operators and narrow operators with respect to Daugavet centers. Visn. Khark. Univ., Ser. Mat. Prykl. Mat. Mekh. 931, No. 62, 5-19 (2010).

[4] R. Deville, G. Godefroy, V. Zizler. Smoothness and renormings in Banach spaces. Pitman Monographs and Surveys in Pure and Applied Mathematics. 64. Harlow: Longman Scientific \& Technical. New York: John Wiley \& Sons, Inc. (1993).

[5] M. Fabian, P. Habala, P. Hásek, V. Montesinos, V. Zizler. Banach space theory: the basis for linear and nonlinear analysis. Springer (2011).

[6] M. I. Kadets, Spaces isomorphic to a locally uniformly convex space. Izv. Vyssh. Uchebn. Zaved. Mat., 1959, no. 6, 51-57 (1959).

[7] V. Kadets, M. Martín, J. Merí, D. Werner. Lushness, numerical index 1 and the Daugavet property in rearrangement invariant spaces. Canad. J. Math. 65, 331348 (2013).

[8] V. Kadets, M. Martín, J. Merí, D. Werner. Lipschitz slices and the Daugavet equation for Lipschitz operators. Proc. Amer. Math. Soc. 143, no. 12, 5281-5292 (2015).

[9] V. Kadets, M. Martín, J. Merí, A. PÉrez. Spear operators between Banach spaces. arXiv:1701.02977, 2017. To appear in Lecture Notes in Mathematics.

[10] V. Kadets And V. Shepelska. Sums of SCD sets and their application to SCD operators and narrow operators. Cent. Eur. J. Math. 8, no. 1, 129-134 (2010).

[11] V. M. Kadets, R. V. Shvidkoy, G. G. Sirotkin, D. Werner. Banach spaces with the Daugavet property. Trans. Amer. Math. Soc. 352, no. 2, 855-873 (2000).

[12] V. M. Kadets, R. V. Shvidkoy, D. Werner. Narrow operators and rich subspaces of Banach spaces with the Daugavet property. Studia Math. 147, 269-298 (2001).

[13] M. Martín and T. OikhberG. An alternative Daugavet property. J. Math. Anal. Appl. 294, no. 1, 158-180 (2004). 
[14] W. Schachermayer The sum of two Radon-Nikodym-sets need not be a RadonNikodym-set. Proc. Amer. Math. Soc. 95, 51-57 (1985).

(Kadets)

School of Mathematics and Informatics,

V. N. Karazin Kharkiv National University, Pl. Svobody 4, 61022 Kharkiv, Ukraine.

ORCID: 0000-0002-5606-2679

E-mail address: v.kateds@karazin.ua

(Pérez)

Departamento de Matemáticas,

UNiversidad DE MURCIA,

30100 Espinardo (Murcia), Spain.

ORCID: 0000-0001-8600-7083

E-mail address: antonio.perez7@um.es

(Werner)

Department of Mathematics,

Freie Universität Berlin,

Arnimallee 6, D-14 195 Berlin, Germany.

ORCID: 0000-0003-0386-9652

E-mail address: werner@math.fu-berlin.de 